\title{
Basal septal hypertrophy in patients with hypertension: a non-invasive assessment of segmental myocardial work with left ventricular pressure-strain relations
}

\author{
DFilip Lončarič ${ }^{*}$, \\ (D)Maciej Marciniak², \\ DJoao Filipe Fernandes ${ }^{2}$, \\ (i)Loredana Nunno ${ }^{3}$, \\ (i)Laura Sanchis ${ }^{3}$, \\ (iD)Bart Bijnens ${ }^{4}$, \\ (D) Marta Sitges ${ }^{3}$
}

IInstitute of Biomedical Research August Pi Sunyer (IDIBAPS), Barcelona, Spain ${ }^{2}$ Kings College, Department of Biomedical Engineering and Imaging Sciences, London, United Kingdom

${ }^{3} \mathrm{Hospital}$ Clinic, Cardiovascular Institute, Barcelona, Spain

${ }^{4}$ University Pompeu Fabra, Department of Information Technology and Communications, Barcelona, Spain

RECEIVED:

October 26, 2018

ACCEPTED:

November 5, 2018

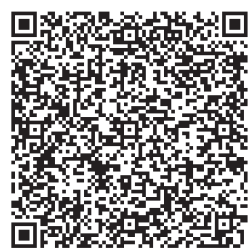

KEYWORDS: myocardial work, basal septal hypertrophy, hypertension, speckle-tracking. CITATION: Cardiol Croat. 2018;13(11-12):411-412. | https://doi.org/10.15836/ccar2018.411

*ADDRESS FOR CORRESPONDENCE: Filip Lončarić, Institute of Biomedical Research August Pi Sunyer (IDIBAPS), Barcelona, Spain / Phone: +385-91-2220-480 / E-mail: Ioncaric.filip@gmail.com

ORCID: Filip Lončarić, https://orcid.org/0000-0002-7865-1108 •Maciej Marciniak, https://orcid.org/0000-0002-4277-7446 Joao Filipe Fernandes, https://orcid.org/0000-0002-4621-1837 • Loredana Nunno, https://orcid.org/0000-0001-5448-968X Laura Sanchis, https://orcid.org/0000-0003-2516-8953•Bart Bijnens, https://orcid.org/0000-0003-3130-6937

Marta Sitges, https://orcid.org/0000-0003-1300-4732

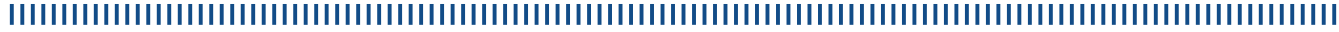

Background: A subgroup of patients with chronic hypertension develop basal septal hypertrophy (BSH). Non-invasive left ventricular (LV) pressure estimates and speckle-tracking deformation curves can be used to quantify myocardial work $(\mathrm{MW})^{1}$. Incorporation of afterload into deformation analysis demonstrates a potential advantage over isolated global longitudinal strain (GLS). The aim is to assess segmental MW indices of the septum in hypertensive patients.

Patients and Methods: An echocardiogram and cuff blood pressure measurement were prospectively performed on $115 \mathrm{pa}-$ tients with hypertension. The interventricular septum was measured in parasternal long axis and 4-chamber (4C) views. LV speckle-tracking was performed in 4C, 2C and 3C views. Myocardial work index (MWI), constructive work (CW), wasted work (WW), and work efficiency (WE) were calculated between mitral valve closing and opening. BSH was defined by having both a positive visual assessment of an abrupt change in septal thickness in $4 \mathrm{C}$ view and a basal septal-mid septal ratio $\geq 1.4$ Results: BSH was present in 18\% ( $\mathrm{n}=21)$ of the cohort. Patients with BSH had higher systolic blood pressure at presentation There was no group difference in cavity dimensions, LV ejection fraction, LV GLS, global MWI. CW or WW. The basal inferoseptum and anteroseptum were significantly thicker in patients with BSH (Figure 1). The hypertrophy was related to a decrease of longitudinal strain (LS) in the inferoseptum, but not in the anteroseptum. The segmental MWI and CW were significantly reduced in the inferoseptum of patients with $\mathrm{BSH}$, whereas there was no difference in WW or WE. No differences in MW indices were notable in the basal anteroseptum (Table 1). There was a pronounced gradient of LS in both groups, with a decrease from apex to base. The averaged LS of the six basal segments was significantly lower in patients with BSH, with no differences in the mid or apical segments (Figure 2).

Conclusion: Basal segments are first affected in chronic exposure to increased afterload, resulting in a gradient of LS. BSH in hypertension indicates a more advanced functional impairment of the LV with further decrease in basal segment function. In this setting the basal inferoseptum is most affected, demonstrating a significant decrease in work performed between mitral valve closing and opening.

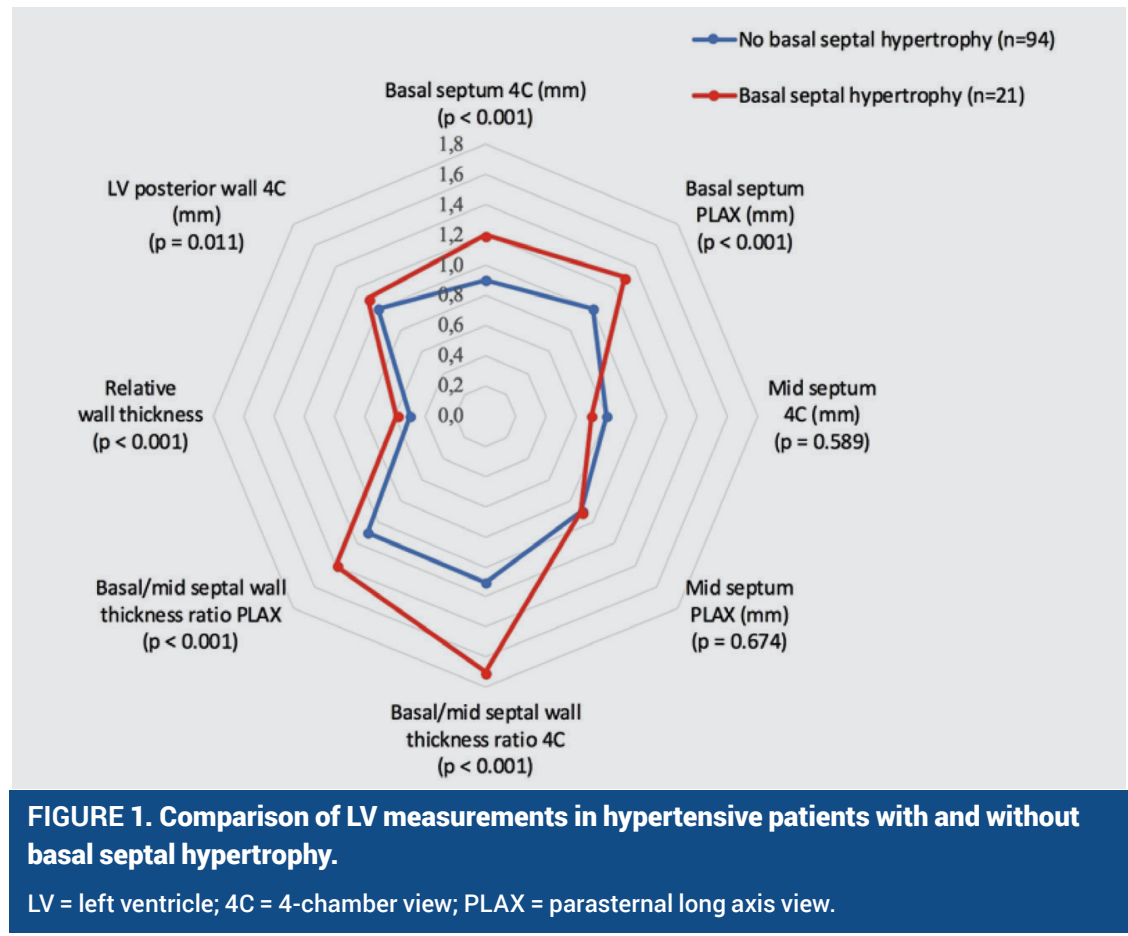


TABLE 1. Global and segmental longitudinal strain and myocardial work indices.

\begin{tabular}{llll} 
& Patients without BSH (n=94) & Patients with BSH (n=21) & P value \\
\hline LV GLS, \% & $-21.8 \pm 2.6$ & $-20.8 \pm 2.5$ & 0.108 \\
\hline Global MWI, mmHg\% & $2631 \pm 457$ & $2654 \pm 426$ & 0.833 \\
\hline Global CW, mmHg\% & $2852 \pm 486$ & $2906 \pm 398$ & 0.638 \\
\hline Global WW, mmHg\% & $64(42,88)$ & $58(38,101)$ & 0.643 \\
\hline Basal inferoseptal LS, \% & $-15.00 \pm 2.84$ & $-12.05 \pm 2.65$ & $<0.001$ \\
\hline Basal inferoseptal MWI, mmHg\% & $1724(1516,2045)$ & $1441(1181,1519)$ & $<0.001$ \\
\hline Basal inferoseptal CW, mmHg\% & $1881(1580,2166)$ & $1500(1324,1747)$ & $<0.001$ \\
\hline Basal inferoseptal WW, mmHg\% & $40(14,92)$ & $16(5,78)$ & 0.173 \\
\hline Basal anteroseptal LS, \% & $-17.76 \pm 3.40$ & $-17.88 \pm 4.37$ & 0.892 \\
\hline Basal anteroseptal MWI, mmHg\% & $2084 \pm 500$ & $2214 \pm 518$ & 0.288 \\
\hline Basal anteroseptal CW, mmHg & $2324 \pm 501$ & $2482 \pm 513$ & 0.197 \\
\hline Basal anteroseptal WW, mmHg\% & $41(11,74)$ & $36(5,130)$ & 0.939 \\
\hline
\end{tabular}

$\mathrm{BSH}$ = basal septal hypertrophy; $\mathrm{LV}$ = left ventricle; $\mathrm{GLS}$ = global longitudinal strain; $\mathrm{MWI}$ = myocardial work index; $\mathrm{CW}$ = constructive work; $\mathrm{WW}$ = wasted work.

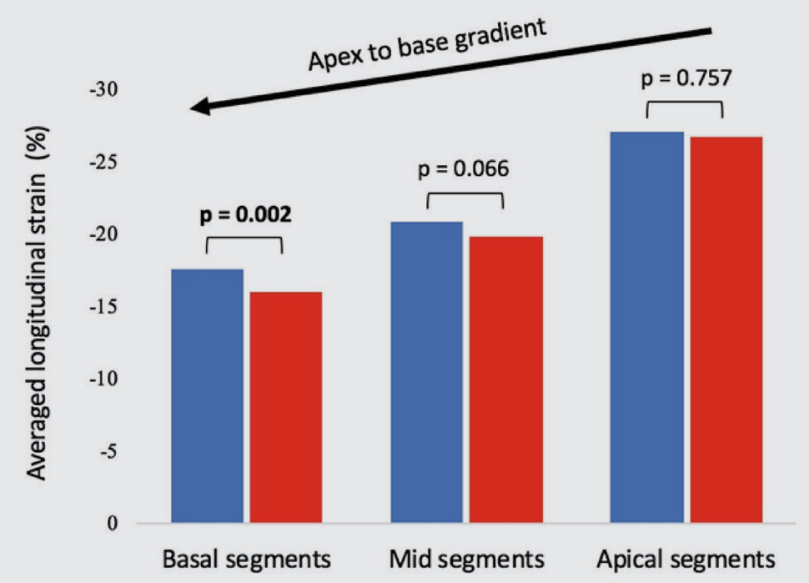

- No basal septal hypertrophy ( $\mathrm{n}=94) \quad$ Basal septal hypertrophy $(\mathrm{n}=21)$

FIGURE 2. Averaged segmental longitudinal strain of basal, mid and apical levels in hypertensive patients.

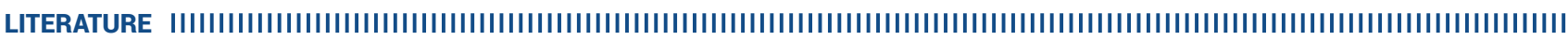

1. Hubert A, Le Rolle V, Leclerca C, Galli E, Samset E, Casset C, et al. Estimation of myocardial work from pressure-strain loops analysis: an experimental evaluation. Eur Heart J Cardiovasc Imaging. 2018 Feb 26. [Epub ahead of print]. https://doi.org/10.1093/ehjici/jey024 